\title{
Pauli Principle, Inflation and Simple Statistical Treatment of Free-Fermions
}

\author{
Angelo Plastino, ${ }^{1,2,3}$, Mario Carlos Rocca1,2,4, Gustavo Ferri ${ }^{5}$ \\ ${ }^{1}$ Departamento de Física, Universidad Nacional de La Plata, La Plata, Argentina \\ ${ }^{2}$ Consejo Nacional de Investigaciones Científicas y Tecnológicas (IFLP-CCT-CONICET)-C. C. 727, La Plata, Argentina \\ ${ }^{3}$ SThAR-EPFL, Lausanne, Switzerland \\ ${ }^{4}$ Departamento de Matemática, Universidad Nacional de La Plata, La Plata, Argentina \\ ${ }^{5}$ Fac. de C. Exactas-National University La Pampa, Peru y Uruguay, Santa Rosa, La Pampa, Argentina \\ Email: angeloplastino@gmail.com
}

How to cite this paper: Plastino, A., Rocca, M.C. and Ferri, G. (2020) Pauli Principle, Inflation and Simple Statistical Treatment of Free-Fermions. Journal of High Energy Physics, Gravitation and Cosmology, 6, 443-449. https://doi.org/10.4236/jhepgc.2020.63034

Received: June 23, 2020

Accepted: July 19, 2020

Published: July 22, 2020

Copyright $\odot 2020$ by author(s) and Scientific Research Publishing Inc. This work is licensed under the Creative Commons Attribution International License (CC BY 4.0).

http://creativecommons.org/licenses/by/4.0/

\begin{abstract}
We study the dependence of the of microstates number (for free fermionsbosons) as a function of the volume-size in quantum statistics and fermions, and show then that fermions can not be accommodated in arbitrarily small volumes $V$. A minimum $V=V_{\min }$ for that purpose is determined. Fermions can not exist for $V<V_{\min }$. This fact might have something to do with inflation. More precisely, in order to accommodate $N$ fermions in a Slater determinant, we need a minimum radius, which is a consequence of the Pauli principle. This does not happen for bosons. As a consequence, extrapolating this statistical feature to a cosmological setting, we are able to "predict" a temperature-value for the final-stage of the inflationary period. This value agrees with current estimates.
\end{abstract}

\section{Keywords}

Microstates's Number $\Omega$, Fermions, Bosons

\section{Introduction}

Cosmic inflation is a widely accepted phenomenon. The detailed particle physics mechanism responsible for inflation is unknown. The basic inflationary paradigm is accepted by most physicists, as a number of inflation model predictions have been confirmed by observation. The inflationary epoch lasted from $10^{-36}$ seconds after the conjectured Big Bang singularity till about $10^{-32}$ seconds after the singularity [1] [2] [3]. Here we will develop a statistical micro-canonical argument that seems to indicate that the Pauli principle might perhaps have played 
some kind of role in the inflation process. Our protagonist is the number of micro-states (multiplicity) $\Omega$ for $N$ particles of energy $E$ enclosed in a volume $V$. We will see that, in the case of fermions, $V$ can not be arbitrarily small. This observation motivates a hopefully attractive interpretation of cosmic inflation.

\section{Multiplicities $\Omega$}

\subsection{Classical}

The multiplicity $\Omega(E, V, N)$ of an $N$-particle, mono-atomic, ideal gas with energy $E$ and volume $V$ is the product of the number of cells of volume $\delta s^{3}$ available in position space (that equals $V / \delta s^{3}$ ) and the number of cells available in momentum space [4]. The volume of momentum space through which the system may move is the p-volume $(2 \mathrm{e} \pi / 3 N)^{3 N / 2} 3 N R^{3 N-1} \delta p$ of a one-cell-wide shell of radius $R=\sqrt{2 m E}$. As a consequence, the number of permissible momentum-space cells in this shell is (e is the basis of natural logarithms)

$$
V_{\text {p-shell }}=(2 \mathrm{e} \pi / 3 N)^{3 N / 2} \sqrt{3 N}(2 m E / 3 N)^{(3 N-1) / 2}\left(\delta p / \delta p^{3 N}\right),
$$

so that $[4]$

$$
\Omega(E, V, N)=(V / \delta V)^{N}(2 \mathrm{e} \pi)^{3 N / 2} \sqrt{3 N}(2 m E / 3 N)^{(3 N-1) / 2}\left(\delta p / \delta p^{3 N}\right) .
$$

After some lengthy considerations, the author of [4] [pp. 56-57] [Eqs. (3.8)(3.15)] rephrases the above relations as

$$
\Omega(E / N, V, 1)=V(E / N)^{3 / 2}\left(4 \mathrm{e} \pi m / 3 h^{2}\right)^{3 / 2},
$$

an equation to be discussed below.

\subsection{Fermions}

It has been known since at least 1925 that quantum mechanics includes, among its tenets, the following tree conditions: 1) phase space cells have a size determined by Planck's constant $h, 2$ ) the energy, momenta, and other dynamical properties of an isolated system are quantized, and 3) for the purpose of determining multiplicity, identical particles are, indistinguishable from one another. Thus, (3) gives the multiplicity $\Omega$ of an ideal gas composed of $N$ distinguishable particles that occupy volume $V$ and share an energy $E$.

If $\Omega(E, V, N)$ is the multiplicity of an ideal gas composed of $N$ distinguishable particles with total energy $E$ and placed in a volume $V$, it follows that $\Omega(E / N, V, 1)$ should be the multiplicity of just a single particle of ideal gas, characterized by $E / N$ and, and occupying volume $V$. This particle composes a larger system of $N$ distinguishable particles. A single particle of an ideal gas occupies $\Omega(E / N, V, 1)$ equally probable microstates. Accordingly,

$$
\Omega(E, V, N)=[\Omega(E / N, V, 1)]^{N} .
$$

A crucial insight is here gotten: the number of cells that can be occupied by a single particle of ideal gas must be independent of whether that particle is itself 
distinguishable or indistinguishable from the other particles that compose the gas system and if indistinguishable whether fermion or boson. A system of $N$ distinguishable particles of ideal gas may occupy $[\Omega(E / N, V, 1)]^{N}$ equally probable microstates [4].

Focus attention now upon an ideal Fermi gas. Let us call $n$ the number of cells that can be occupied by a single fermion $E / N$. We have $n / N \geq 1$. According to (3) we have

$$
n=V(E / N)^{3 / 2}\left(4 \mathrm{e} \pi m / 3 h^{2}\right)^{3 / 2},
$$

with

$$
V=\frac{4}{3} \pi r^{3} .
$$

We face the problem of filling $n$ cells with $N \leq n$ identical fermions. Thus the multiplicity becomes [4]

$$
\Omega=\frac{n !}{N !(n-N) !} .
$$

This multiplicity derived above is the result of the so-called average energy approximation. According to this approximation, each gas particle possesses the same energy $E / N$ for all fermions in the system. That the average energy approximation produces the exact multiplicity when identical particles are considered distinguishable. The average energy approximation has been extensively used and produces quite reasonable results [4].

We approximate the Gamma function using the Stirling recipe

$$
\Gamma(z)=\sqrt{2 \pi} z^{z-\frac{1}{2}} \mathrm{e}^{-z},
$$

and find $(\Theta$ is Heaviside's step function)

$$
\Omega=\frac{e e^{(n+1) \ln (n+1)-\Theta(n-N+1)(n-N+1) \ln (n-N+1)}}{\sqrt{2 \pi}(N+1)^{(N+1)}},
$$

and introduce the notationally simplifying definition (change of variables from $r$ to $s$ )

$$
s=\left(\frac{4 \pi}{3 N}\right)^{\frac{1}{3}}\left(\frac{E}{N}\right)^{\frac{1}{2}}\left(\frac{4 \pi e m}{3 h^{2}}\right)^{\frac{1}{2}} r,
$$

so that

$$
\Omega=\frac{\mathrm{e}^{\left(1+N s^{3}\right) \ln \left(1+N s^{3}\right)} \mathrm{e}^{-\Theta\left[N s^{3}-(N-1)\right]\left[N s^{3}-(N-1)\right] \ln \left[N s^{3}-(N-1)\right]}}{\sqrt{2 \pi}(N+1)^{(N+1)}} .
$$

Of course, it must be

$$
\Omega \geq 1 ; \quad \ln (\Omega) \geq 0
$$

From (11) one gets 


$$
\begin{aligned}
\ln (\Omega)= & \left(1+N s^{3}\right) \ln \left(1+N s^{3}\right)-\Theta\left[N s^{3}-(N-1)\right]\left[N s^{3}-(N-1)\right] \\
& \times \ln \left[N s^{3}-(N-1)\right]+1-\frac{1}{2} \ln (2 \pi)-(N+1) \ln (N+1),
\end{aligned}
$$

and we ask for a possible vanishing of $\ln (\Omega)$. This is the critical novel issue that we address in this paper.

Since $N \gg 1$ we are led to answer with the relation

$$
\frac{\ln (\Omega)}{N} \simeq s^{3} \ln \left(s^{3}\right)-\Theta\left(s^{3}-1\right)\left(s^{3}-1\right) \ln \left(s^{3}-1\right)=0,
$$

that has two possible solutions, namely,

$$
s=0, \quad s=1 .
$$

Figure 1 shows that the multiplicity $\Omega$ would be negative for $0 \leq s \leq 1$, which is absurd. We gather that the system is subjected to a kind of "phase transition" at $s=0$ and forced to "jump" to $s=1$. One could putatively associate this jump to a sort of inflation-phenomenon motivated by the Pauli principle that would forbid $N$ fermions to be accommodated in a too small volume $V$. A critical "accommodating" volume is reached at $s=1$. Emphasize that this happens for free fermions micro-canonically described. Equilibrium prevails.

The paper could well finish here. However, it is too tempting to extrapolate a bit further. We do this below, after discussing Bosons.

\subsection{Bosons}

The multiplicity is, for $n$ microstates and Nbosons [4],

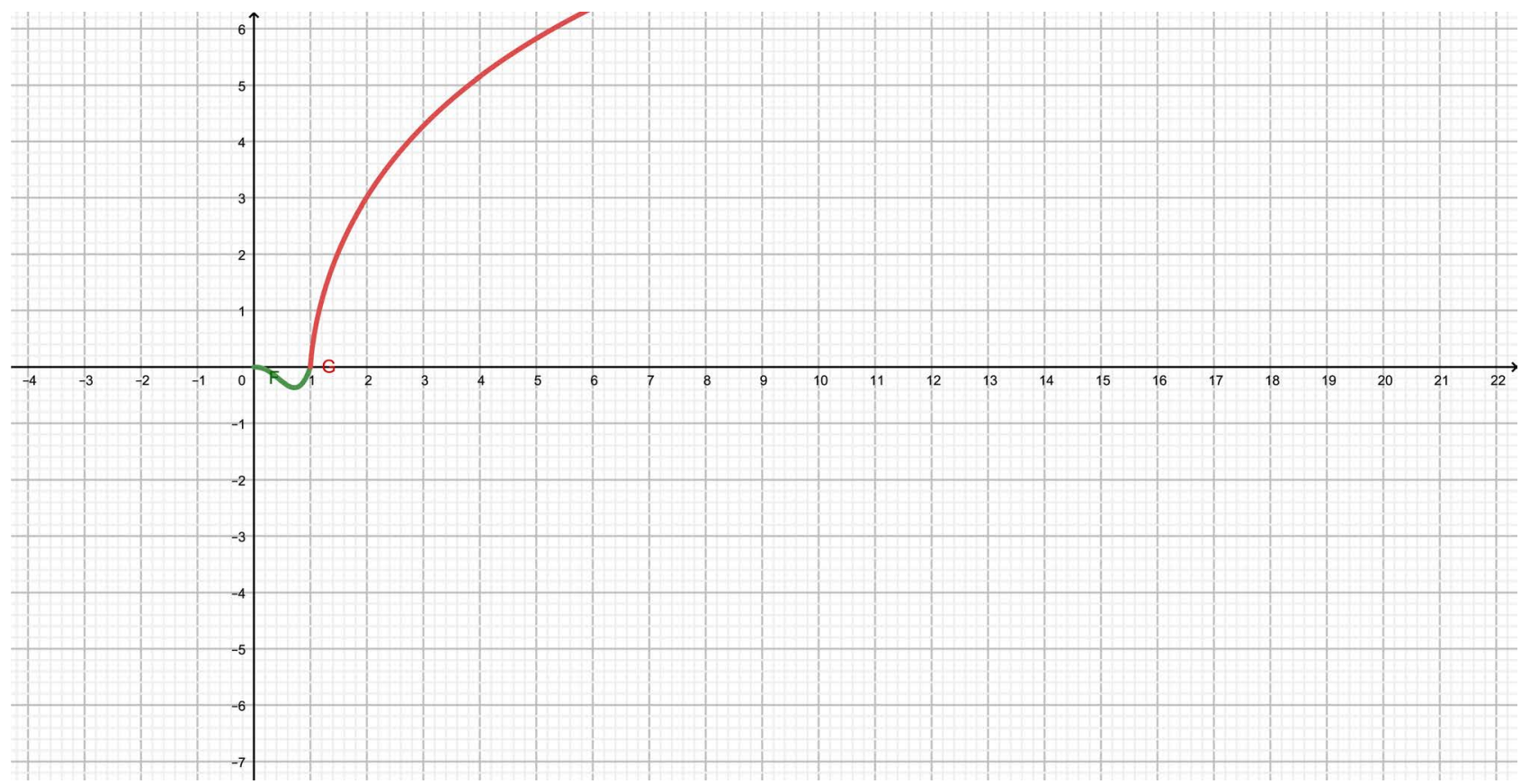

Figure 1. FERMIONS: The logarithm of the number of microstates $\ln \Omega$ versus the $N$ fermions' size-indicator $s$. Note the sudden size-increase at the origin, which one might be willing to associate to inflation, that in turn would be motivated by the Pauli principle. 


$$
\Omega=\frac{(N+n-1) !}{N !(n-1) !} .
$$

The counting performed above is structurally identical to counting the number of distinct manners of ordering a set of $N$ identical balls and $n-1$ identical white dividers. If placed in a row, these $n-1$ dividers separate our $N$ balls into $n$ ordered (distinct) groups [4].

Thus, things are quite different for bosons, not subjected to the exclusion principle. The above $\Omega$, for $N$ bosons of mass $m$ contained in $V$ with total energy $E$ will yield [4]

$$
\Omega=\frac{\mathrm{e}^{\left(N+N s^{3}\right) \ln \left(N+N s^{3}\right)} \mathrm{e}^{-\left(N s^{3}\right) \ln \left(N s^{3}\right)}}{\sqrt{2 \pi} N^{N}},
$$

with $s$ given by (2).

$$
\ln (\Omega)=\left(N+N s^{3}\right) \ln \left(N+N s^{3}\right)-\left(N s^{3}\right) \ln \left(N s^{3}\right)-\frac{1}{2} \ln (2 \pi)-N \ln (N) .
$$

From (7) it follows that, in the spirit of last Subsection,

$$
\frac{\ln (\Omega)}{N} \simeq\left(1+s^{3}\right) \ln \left(1+s^{3}\right)-\Theta(s) s^{3} \ln \left(s^{3}\right)=0,
$$

whose solution is

$$
s=0,
$$

as illustrated in Figure 2.

\subsection{A Putative Interpretation}

What might have happened when fermions began to emerge at the Big-bang?

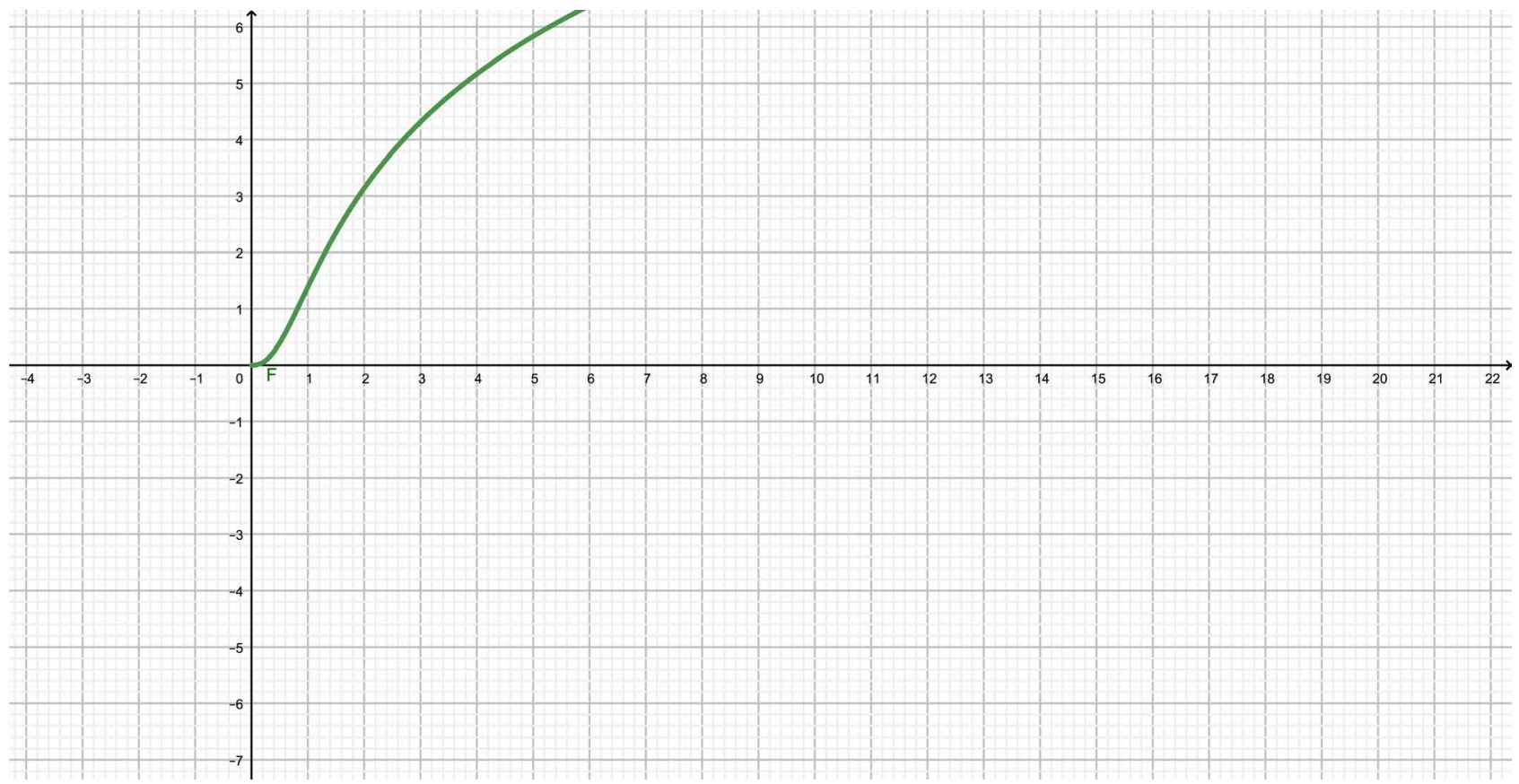

Figure 2. $\ln \Omega$ versus $s$ for bosons. Nbosons can be accommodated at the origin. Remaining details are as in Figure 1 . 
A tiny fraction of a second after the singularity, some fermions began to emerge, out of a quark-gluon plasma [5], $N$ of them, at a tiny region of size $V$, whose total fermion-energy was $E$ (this variables determine a microcanonical ensemble). This region had to "explode" in order to accommodate them (transition from $s=0$ to $s=1$ above).

If we accept Siegel's estimate for the Universe's radius at the end of the inflation period of $r=r_{0}=17 \mathrm{~cm} \mathrm{[5],} \mathrm{then,} \mathrm{from} \mathrm{Equation} \mathrm{(11),} \mathrm{one} \mathrm{gathers} \mathrm{that}$ one has for $N$, when $s=1$,

$$
N=\frac{4 \pi}{3}(m c)^{\frac{3}{2}}\left(\frac{4 \pi \mathrm{e} m}{3 h^{2}}\right)^{\frac{3}{2}} r_{0} .
$$

If we set $\frac{E}{N}=m c^{2}$ (lower bound for $E$ ), with $m=1.6 \times 10^{-27} \mathrm{~kg}$ the baryon mass, then the fermion number becomes $N=1.15 \times 10^{48}$ at the critical volume referred to above, a very small number compared to today's estimate of $10^{79}$. Of course, radiation, neutrinos and plasma predominate at this stage [5].

\subsection{Temperature at the End of the Inflationary Period}

The entropy at the end of the inflationary period $(s=1)$ reads

$$
\left.S\right|_{s=1}=\left.k_{B} \ln \Omega\right|_{s=1},
$$

with $k_{B}$ the Boltzmann constant. For the temperature $T$ we have

$$
\left.\frac{1}{T}\right|_{s=1}=\left.\frac{\partial S}{\partial E}\right|_{(V, N, s=1)},
$$

or

$$
\left.\frac{1}{T}\right|_{s=1}=\left.\left.\frac{\partial S}{\partial s^{3}}\right|_{(V, N, s=1)} \frac{\partial s^{3}}{\partial E}\right|_{(V, N, s=1)} .
$$

More explicitly, one has

$$
\left.\frac{1}{T}\right|_{s=1}=\frac{2 \pi r_{0}^{3} k_{B} m^{2} c}{h^{3}}\left(\frac{4 \pi \mathrm{e}}{3}\right)^{\frac{3}{2}} \ln (1+N) .
$$

Using the appropriate values for $k_{B}$, $h$, etc., we obtain $T=3.9 \times 10^{18}$ Kelvin degrees, which agrees with the value estimated in reference [6]. Note that in our case we are advancing a statistical prediction.

\section{Conclusion}

We have here introduced a simple, micro-canonical statistical argumentation purporting to show that, on account of Pauli's principle, $N$ fermions can not be accommodated in an arbitrarily small volume $V$, as bosons can. We statistically determined a minimum critical volume-value for $N$ fermions. One is then tempted to extrapolate the above findings to a cosmological setting and predict a numerical value for the temperature prevailing at the end of the inflationary pe- 
riod. Our prediction agrees with current estimates.

\section{Conflicts of Interest}

The authors declare no conflicts of interest regarding the publication of this paper.

\section{References}

[1] The Kavli Foundation (2014) "First Second of the Big Bang". How the Universe Works, Discovery Science.

[2] Tyson, A., de Grasse, N. and Goldsmith, D. (2004) Origins: Fourteen Billion Years of Cosmic Evolution. W. W. Norton \& Co., New York, pp. 84-85.

[3] Tsujikawa, S. (2003) Introductory Review of Cosmic Inflation. https://arxiv.org/abs/hep-ph/0304257

[4] Lemons, D.S. (2014) A Student's Guide to Entropy. Cambridge University Press, Cambridge.

[5] Siegel, E. (2017) FORBES, Starts with a Bang. https://www.forbes.com/sites/startswithabang/2017/03/24/how-big-was-the-univers e-at-the-moment-of-its-creation/\#43e61dcf4cea

[6] Jose F. Garcia.

http://lahoracero.org/fisica-de-particulas-big-bang-temperatura-energia-y-lhc/ 